To Remove Please Registered With http://www.pdf-converter-software.com

\title{
The role of the autonomic reflex tests in arrhythmology
}

\author{
Ph.D. Thesis
}

Attila Makai MD.

Szeged

2011. 
To Remove Please Registered With http://www.pdf-converter-software.com

\author{
University of Szeged \\ $2^{\text {nd }}$ Department of Internal Medicine and Cardiology Cenre \\ Doctoral School of Clinical Medicine - Clinical and Experimental Cardiovascular Science
}

The role of the autonomic reflex tests in arrhythmology

Ph.D. Thesis

Dr. Attila Makai

Tutor: Prof. Dr. László Rudas

Szeged

2011. 


\section{TOPIC RELATED ARTICLES}

1. Makai A, Sztriha L, Vörös E, et al. Haemodynamic instability induced by carotid stent implantation. Orv Hetil 2006;147:2515-2521.

2. Makai A, Korsós A, Makra P et al. Spontaneous baroreflex sensitivity and heart rate turbulence parameters: parallel responses to orthostasis. Clin Auton Res 2008;18:74-79.

3. Makai A, Gallardo R, Traykov V et al. Converging methods in the assessment of sympathetic baroreflex sensitivity. Europace. 2010 12:574-578.

4. Makai A, Csillik A, Csanádi Z et al. Unusual case of orthostatic tachycardia. Orv Hetil 2007;148:77-80.

5. Makai A, Tahin T, Simor T et al. Postural tachycardia syndrome Orv Hetil 2005;146:515-20.

6. Sztriha L, Vörös E, Sas K et al. Favorable early outcome of carotid artery stenting without protection devices. Stroke 2004;35:2862-6.

7. Avramov K, Sztriha L, Makai A et al. Mechanism of cough syncope Orv Hetil 2004;145:1625-1627.

8. Makai A, Sághy L, Rudas L et al. Wenckebach AV block, as part of vasovagal syncope Orv Hetil 2002;143:2351-2353.

9. Makai A, Zöllei E, Halmai L, et al. Atrial fibrillation provoked by Valsalva maneuver Orv Hetil 1999;140:2811-2812

\section{TABLE OF CONTENTS}

1. Introduction

2. Aims and hypotheses

3. Materials and Methods

4. Results

5. Discussion (Interpretations and Conclusions)

6. Limitations

7. Respective findings and future clinical implications 


\section{INTRODUCTION}

Cardiac rhythm is under strong autonomic regulation. The importance of the autonomic influences in the genesis of supraventricular- and ventricular arrhythmias, and in sudden death has been long recognized. Autonomic regulation is however not regularly studied in clinical arrhythmology. Notable exceptions are the group of neutrally mediated syncope. Abnormal reflexes may occur during various medical interventions, prompting debate regarding the indication of temporary pacemaker therapy. Such intervention is carotid artery dilation and stenting, where frightening episodes of longer lasting bradycardia have been reported Temporary pacemaker therapy however carries certain risk. Methods to assess the real hazard of the neutrally mediated reactions, thus minimalizing the iatrogenic complications, are needed.

Reflex abnormalities detectable in post-myocardial infarction state and congestive heart failure could be important for the arrhythmologists. Certain markers of the autonomic regulation do have the capacity to predict short-, and long time adverse outcome including sudden cardiac death. Therefore an abnormal parameter may help to define patient subpopulations that could have benefit of more aggressive preventive therapy, such as ICD implantation. In spite of their potential predictive capacity, autonomic markers have not yet qualified to serve as ICD implantation criteria. The most promising parameter, the cardiovagal baroreflex sensitivity (BRScv), requires arterial pressure recording. Predictive power of BRScv was demonstrated in the convincing ATRAMI trial, however in that study intra-arterial arterial pressure recording, and pharmacological baroreflex challenge (i.e. phenylephrine injection) was used. Neither of them is suitable for routine clinical investigations. Although noninvasive continuous blood pressure recorders are now available, and spontaneous, rather than pharmacological tests are in use, the ATRAMI-based cut-off values have not yet been validated with the noninvasive methods. Recently, a pair of promising new risk-predictor parameters, the heart rate turbulence onset (HRT onset)-, and turbulence slope (HRT slope) has emerged. These parameters are based on the RR interval fluctuations subsequent to a premature ventricular complex (PVC). Calculation of the HRT parameters does not require complicated instruments or software. These parameters could be easily derived for Holter recordings containing PVC-s in sufficient number. Nevertheless HRT interpretation needs further refinements. Physiological considerations suggest that Holter based HRT parameters are closely related to BRScv. Therefore HRT, - similar to the BRScv, - could be subject of external influences. Delineation of these influences may help to put the Holter-based information into context.

Cardiovagal parameters could be easily assessed. Pulse intervals are readily available and analyzable on ECG recordings, and commercially available Holter monitors are capable to calculate the well known time-, and frequency domain heart rate variability, (HRV) parameters. It is possible that impaired vagal regulation contribute directly to the adverse outcome however the evidence supporting this theory is mostly circumstantial. In contrast, there is abundant evidence indicating, that in advanced cardiac diseases the uncontrolled sympathetic activation contribute tremendously to the progress of the pathological process. Analyzability of the short-time sympathetic regulation in the clinical practice however is limited. Baroreflex regulation of vasomotor muscle sympathetic nerve activity (MSNA) exhibits well-known abnormalities in conditions, such as congestive heart failure, and essential hypertension. MSNA recording however is an invasive technique and requires special equipment and skill. A simple clinical test for BRSsym is badly needed in clinical cardiology. Such a simple sympathetic baroreflex index, derived from the blood pressure dynamics during the late phases of Valsalva maneuver was recently proposed from the Mayo 
Clinic, by the group of Philip Low. At the same time, arrhythmologists, who studied the genesis of HRT, introduced new parameters based on post extrasystolic blood pressure fluctuations. These "post-extrasystolic blood pressure turbulence slopes" are theoretically analogous to the pressure recovery slopes derived from the recovery phase of Valsalva maneuver. By confirming this analogy we could prove the convergence of the new techniques, allowing unification of methods, terms and concepts in studying various healthy a disease stricken populations.

\section{AIMS AND HYPOTHESES}

The main goal of the thesis is to assess the genesis, modulations, and predictive power of certain noninvasive autonomic markers, which could allow screening the candidates for temporary-, or permanent electrical device therapy.

- Temporary pacemaker therapy is frequently performed in patients undergoing carotid artery stenting. This procedure however presents risk of complications, requires special equipment, and assistance of a specialist. Reducing preventive temporary pacing to a high risk subset of patients could be safe and cost-effective. Therefore our aim was to test the feasibility, and accuracy of noninvasive BRS testing in predicting long, (>3s) pauses, in a cohort of subsequent patients awaiting carotid artery stenting.

- HRT analysis is a new noninvasive tool in assessing autonomic heart rate control, thus predicting adverse outcome, including sudden death. This method has the potential to qualify as a screening tool for ICD implantation among patients with poor systolic cardiac function both with ischemic-, and non-ischemic origin. HRT however is usually derived from Holter tapes, thus the results are modified by unknown external influences. Therefore our aim was to determine the postural influences on HRT parameters.

- Pulse derived, vagally mediated autonomic parameters are extensive used in clinical practice. The sympathetic activity, - which also has a great impact on cardiovascular morbidity, and mortality, - is not readily assessable. Therefore our goal was to assess a new sympathetic baroreflex marker, derived from the arterial pressure recovery subsequent to an episode of a short lasting, paced ventricular tachycardia. Our aim was also to compare this new parameter to another sympathetic baroreflex index, which could be derived from the pressure responses of the Valsalva maneuver.

\section{MATERIALS AND METHODS}

\subsection{Patient populations}

BRScv parameters prior-to-, and during carotid stent implantation were studied in twenty-four consecutive patients (14 male, 10 female). Eleven patients (46\%) had symptomatic stenosis. The presence of hypertension was noted in twenty-one patients (88\%), diabetes in eleven (46\%), hyperlipidemia in seven (29\%), and ischemic heart disease in eight $(33 \%)$ cases. Three patients (13\%) had a history of peripheral arterial disease.

For the study, assessing the relationship between different sympathetic baroreflex parameters, 25 subjects were recruited from patients waiting for cardiac electrophysiological study. Only patients with preserved left ventricular systolic function were selected (ejection fraction $(\mathrm{EF})>50 \%$ ). Patients with diabetes, Parkinson's disease or with a history of excessive alcohol consumption were not considered. 
For the study, where we assessed the postural effects on HRT and BRScv parameters, the participants were recruited from the pacemaker outpatient clinic at University of Szeged. Subjects with ventricular pacemaker lead and predominant sinus rhythm were selected. No patients with sick sinus syndrome, left ventricular dysfunction $(\mathrm{EF}<50 \%)$ or congestive heart failure (NYHA III-IV) were included.

\subsection{Study equipment, monitors}

In all studies, included to the dissertation, continuous ECG monitoring was performed with a bedside Marquette Eagle monitor. ECG channels with high signal-noise ratio were selected, with well discernible positive $\mathrm{R}$ waves. Blood pressure was monitored noninvasively with a Finapress 2300 (Ohmeda) device.

\subsection{Laboratory environment, data acquisition}

The study on the autonomic responses to carotid artery stenting was performed at the Department of the Radiology of the University of Szeged. A 3-minute baseline recording was performed in all patients, prior to interventions. Continuous recording was than performed throughout the whole stent implantation procedure.

The two studies on the mechanisms and modifiers of the ventricular extrasystole / ventricular tachycardia induced hemodynamic responses were performed in the electrophysiology laboratory and the tilt table laboratories of the University of Szeged.

In all of the studies included to the dissertation, analogous biological signals were recorded by DATAQ/WINDAQ ${ }^{\circledR}$ digitalizing system (DATAQ Instruments, Inc., Akron, $\mathrm{OH}, \mathrm{USA}$ ), at $250 \mathrm{~Hz}$ per channel (in the study on sympathetic baroreflex indices), or at 500 $\mathrm{Hz}$ per channel (in the other 2 studies included to the dissertation).

\subsection{Study protocols}

Patients, waiting for carotid artery stent implantation procedure were managed according to the protocol adopted by our team at University of Szeged. (For details see Sztriha L et al Stroke, 2004).

In the study on different sympathetic BRS indices the subjects underwent cardiac electrophysiological diagnostic study before our measurements. Valsalva maneuvers were repeated at $40 \mathrm{mmHg}$ for 12 secs until the first acceptable recording (by length, maintained pressure, and character of blood pressure recording) was obtained. In order to elicit blood pressure drops of similar magnitude to that of the second phase of Valsalva maneuvers, short ventricular runs ( 4 to 6 beats), with a cycle length of $<60 \%$ of the prevailing sinus rhythm were applied.

In the study on postural effects on HRT and BRScv parameters, patients, arriving to the laboratory assumed supine position on the tilt table. Following a few minutes of adaptation, the patients' prevailing average RR intervals were determined. Then with the external pacemaker programmer, a pacing frequency was initiated corresponding to approximately $60 \%$ of the prevailing RR intervals. After the first captured paced beat, the pacemaker frequency was quickly switched back to the original low backup rate. This maneuver was repeated five times. Each captured paced beat was separated by at least 60 uninterrupted sinus cycles. The tilt table was than elevated to 70 degrees head-up right position. In this position another 5-minute ECG and arterial pressure recording was taken. Subsequently the externally paced series of PVC-s was repeated. 


\subsection{Data analysis}

For the off-line analysis of the recordings the Windaq ${ }^{\circledR}$ program, (DATAQ Instruments, Inc., Akron, OH, USA), and theWinCPRS ${ }^{\circledR}$ software (Absolute Aliens Ay, 2000) were used.

From the noise-free and extrasystole-free baseline recordings the parameters of blood pressure and heart rate variability, as well as the markers of cardiovagal baroreflex sensitivity were determined in all included studies. BRScv was characterized by the method of "spontaneous sequences". Spontaneous sequences were defined as three or more cardiac cycles with continuous systolic blood pressure elevation or decrease accompanied by the unidirectional RR-interval changes. The minimum acceptable change in systolic pressure was set at one $\mathrm{mmHg} / \mathrm{cycle}$. Linear regression analysis between systolic blood pressure and RR interval transients were performed for each sequence, and only those with an $R$ value of $\geq 0.8$ were accepted for further analysis. The BRScv was defined as change in the R-R interval per unit change in systolic blood pressure measured in units of $\mathrm{ms} / \mathrm{mmHg}$. Since the slopes of the regressions of increasing (up) and decreasing (down) sequences may differ significantly, in all studies the up-and down sequences were assembled and averaged separately (Figure 1.). At least three spontaneous up and three down sequences were required during the three-minute period in order to accept their average (up BRS and down BRS) as appreciable data.

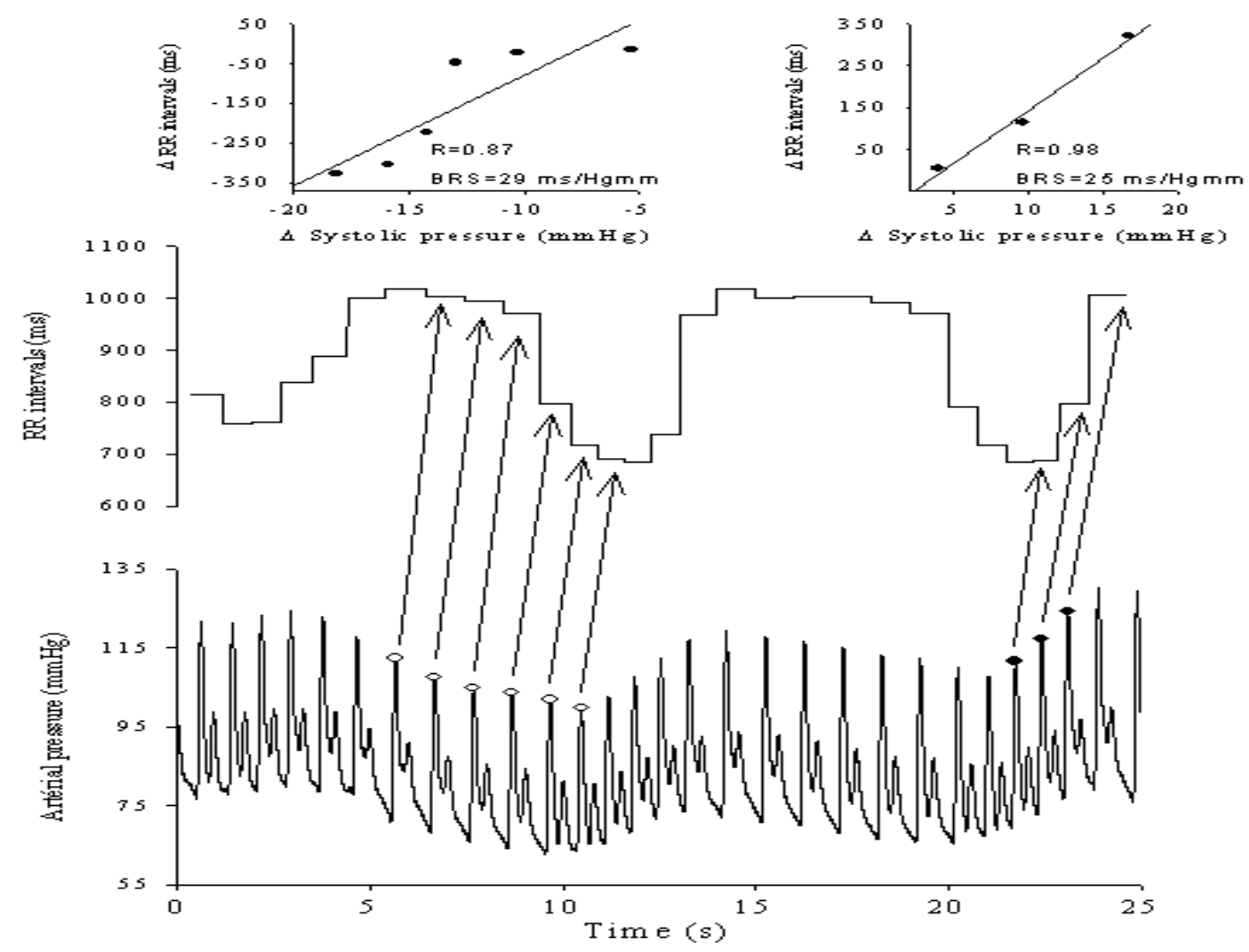

Figure 1. Illustration of the spontaneous up- and down cardiovagal BRS sequences. Open circles represent the down BRS segment, and closed circles represent the up BRS segment of the recording.

In the analysis of the recordings which were taken at the time of stent deployment, duration of the longest induced RR pause was determined. A subgroup was formed on the analogy of carotid sinus hypersensitivity. Patients in the "pathological pause" (PP) subgroup 
exhibited a pause of $\geq 3$ seconds. Data, collected in the subgroup with PP were compared to the subgroup without pathological pauses (WP). The change of the systolic pressure was compared to a baseline, - determined as an average of the last three systolic values prior to stent dilation. The minimum systolic blood pressure value was typically recorded during the first cycle following the pause in the asystolic subgroup.

In the study, comparing sympathetic baroreflex indices, cardiac cycles were defined by RR intervals for both sinus-, and paced NSVT periods. Mean arterial pressure (MAP) was selected for comparison of the different tests. Baseline MAP was determined from 20 cardiac cycles immediately preceding the NSVT and the Valsalva maneuver. The minimum MAP values, and the drops compared to baseline ( $\triangle \mathrm{MAP}$ ) were determined during phase 3 of the Valsalva maneuver and following the paced NSVT series (Figures 2. and 3.). The MAP return times ( $\Delta$ time) were defined as the time elapsing from the onset of the cardiac cycle with the minimum MAP to the onset of the first cycle exceeding the baseline (Figures 2. and 3.). The sympathetic BRS values were determined as the ratio of MAP drop and return time; expressed as $\mathrm{mmHg} / \mathrm{s}$. $\mathrm{SBRS}_{\text {vals }}$ was derived from a single Valsalva maneuver. $\mathrm{SBRS}_{\mathrm{NSVT}}$ was defined as the average of gains; derived from two NSVT series. Within a 15-cycle pressure recording, which followed immediately the NSVT, the maximum slope of 5 consecutive MAP steps was also calculated, and expressed as MAP turbulence slope $\left(\mathrm{MAP}_{\mathrm{TS}}\right)$ in $\mathrm{mmHg} /$ cycle.

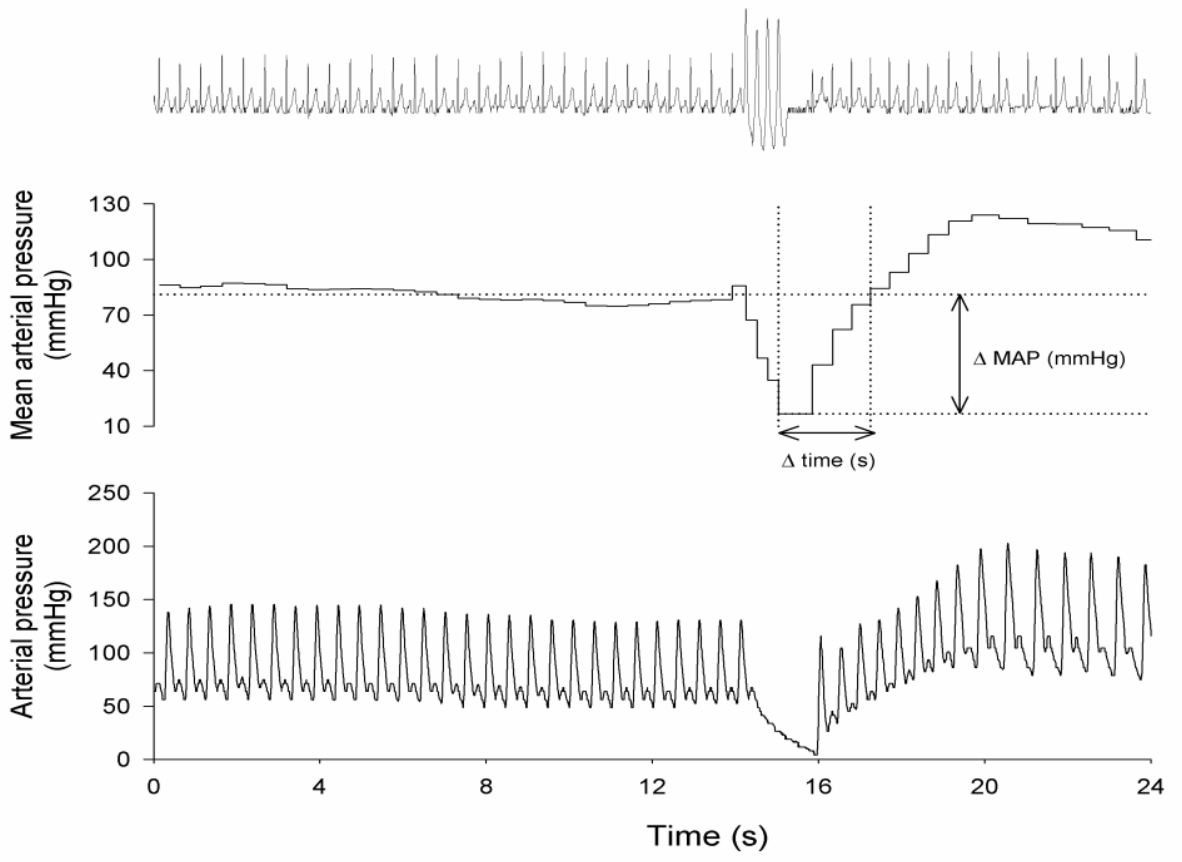

Figure 2. A run of NSVT induces substantial drop in MAP. SBRS $S_{N S V T}$ is defined as the ratio of the $\triangle M A P$ to recovery time. 


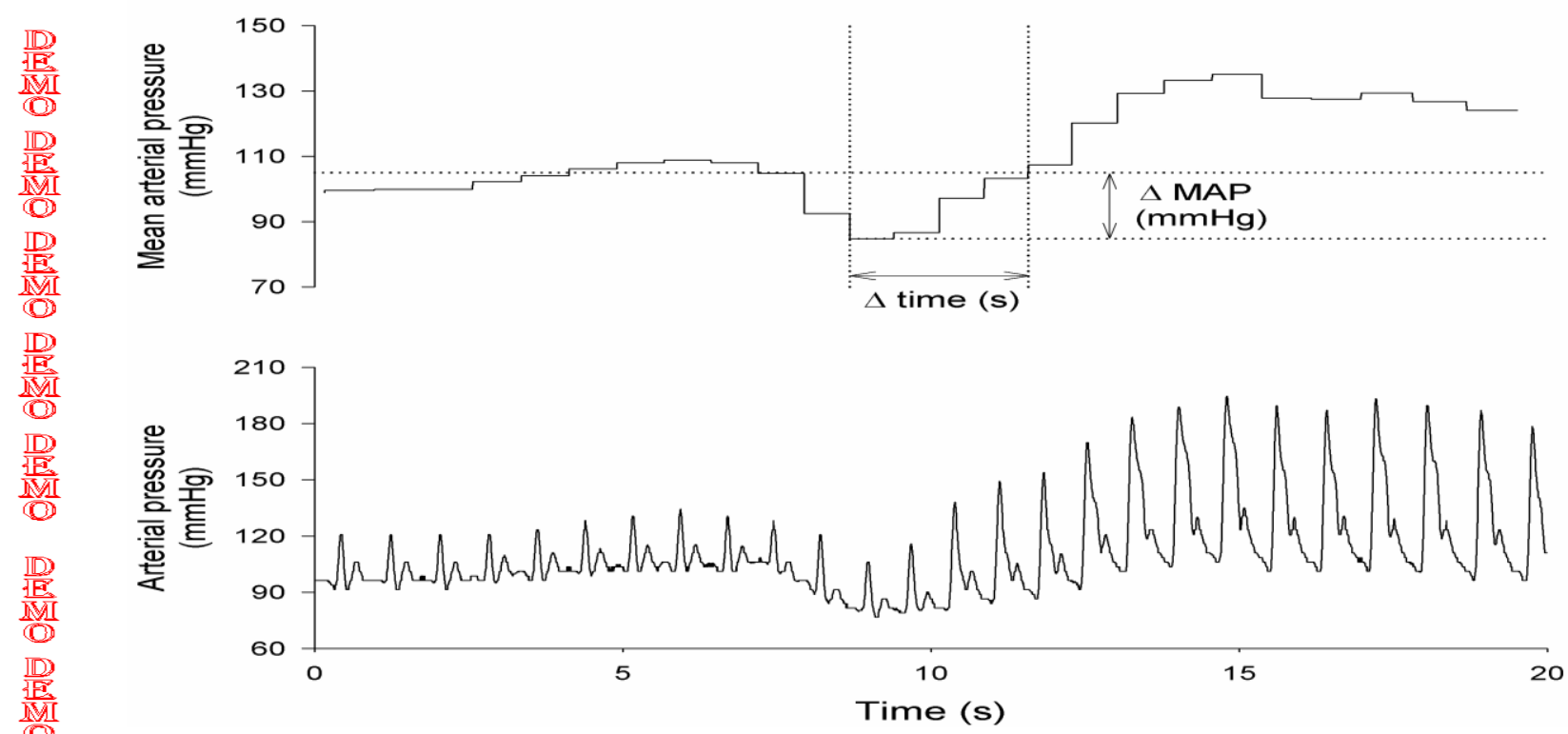

Figure 3. Late phases (late 2, 3 and 4) of a Valsalva manoeuvre. SBRS $S_{\text {vals }}$ is defined as the ratio of the $\triangle M A P$ to recovery time.

In our third study, where relationship of BRS and paced extrasystole induced HRT was assessed, the turbulence onset (TO) was defined as the difference between the mean of the first two RR intervals following the paced PVC and the last two RR intervals before the PVC, divided by the mean of the last two RR intervals before the PVC. Turbulence slope, (TS) was defined as the maximum positive slope of regression line over any sequence of five consecutive sinus-RR intervals within the first 15 cycles after the paced PVC. The average of supine and upright TO and TS parameters were calculated separately for each patient.

\subsection{Statistical analyses}

Unpaired T-test was used for the comparison of the heart-rate variability and baroreflex parameters between the subgroups with-, and without pathological pauses during carotid artery stenting. Comparison of the hemodynamic and reflex responses following paced ventricular tachycardia and Valsalva maneuver were performed by paired $t$ tests. The Wilcoxon Signed Rank test was used for parameters showing skewed distribution. The relationship between the two baroreflex indices and the $\mathrm{MAP}_{\mathrm{TS}}$ was assessed by linear regressions. The agreement between SBRSvals and SBRS ${ }_{\mathrm{NSVT}}$ was also assessed by Bland Altman plot. Relationship between the BRS and HRT parameters were assessed by linear regression. Comparison of supine and upright data was performed using paired $t$ test.

\section{RESULTS}

\subsection{Hemodynamic and reflex responses to carotid artery stenting.}

Pause, exceeding three seconds was seen in seven cases $(29 \%)$, two of which were complicated by syncope. The average duration of the pauses was 7.2 \pm 3.0 seconds (range: $3.7-$ 11.5 seconds). The systolic blood pressure drop in this PP group was $76 \pm 27 \mathrm{mmHg}$, whereas in the group without pause it was $34 \pm 29 \mathrm{mmHg}(\mathrm{p}<0.003)$. Seven $(29 \%)$ patients were given 
atropine during the interventions due to asystole or significant bradycardia. All episodes of pathological pauses terminated quickly with no residual symptoms.

Assessment of baseline recordings was impeded by several factors. The heart rate analysis was precluded in one case by fixed pacemaker rhythm, in one case by atrial fibrillation; in another five cases by frequent ventricular or supraventricular extrasystoles. Motion artifacts precluded the blood pressure analysis in three cases. Some of the technically good records failed to show the minimally required sequence number. Therefore we were able to determine the baroreflex sensitivity index of increasing sequences in twelve cases, while that of decreasing sequences in nine cases. Data of the subgroups are shown in Table 1.

Table 1. Autonomic markers and hemodynamic responses of the PP and no PP subgroups.

\begin{tabular}{lccc}
\hline & "PP" & no "PP" & p \\
\hline Pause (s) & $7.2 \pm 3$ & - & NS \\
MEAN RR (ms) & $788 \pm 70$ & $840 \pm 81$ & NS \\
SDRR (ms) & $28 \pm 10$ & $25 \pm 8$ & NS \\
RMSSD (ms) & $13 \pm 8$ & $14 \pm 5$ & NS \\
UP-BRS (ms/Hgmm) & $3.4 \pm 2$ & $3.3 \pm 2$ & NS \\
DOWN-BRS (ms/Hgmm) & $3.7 \pm 1$ & $4.4 \pm 2$ & NS \\
BASELINE SBP (Hgmm) & $147 \pm 26$ & $154 \pm 31$ & NS \\
MINIMUM SBP (Hgmm) & $71 \pm 31$ & $120 \pm 34$ & $<0.003$ \\
dSBP (Hgmm) & $76 \pm 27$ & $34 \pm 29$ & $<0.003$ \\
\hline
\end{tabular}

There was no statistically significant difference between the HRV and BRS indices in the subgroups.

\subsection{Hemodynamic and reflex responses to short-lasting paced VT and Valsalva maneuver.}

The cardiac electrophysiology study was uneventful in all cases. Based on a preliminary assessment, five recordings were excluded from further analysis; two recordings because of the inability of the subject to perform acceptable Valsalva maneuver and frequent Valsalva induced PVC-s, preventing analysis in three other recordings. Recordings of 20 patients (11 female) entered the final analysis. Their mean age was $57 \pm 17$ years. Seventeen patients suffered from atrioventricular nodal re-entry tachycardia, 1 from paroxysmal atrial flutter, 1 from atrioventricular reentry tachycardia and 1 from right ventricular outflow tract tachycardia. Co-morbidity included mild, controlled hypertension in 13 cases.

The paced NSVT consisted of 5-6 cycles with the driving frequency of $184 \pm 40 / \mathrm{min}$ (range $120-270 / \mathrm{min}$ ). $\triangle$ MAP was slightly but significantly greater following NSVT than during the recovery phase of the Valsalva maneuver $(37 \pm 18$ vs. $45 \pm 17 \mathrm{mmHg}, \mathrm{p}<0.04)$. Accordingly, the recovery time from NSVT was slightly longer ( $4.9 \pm 3$ vs. $6.2 \pm 7 \mathrm{~s}, \mathrm{P}=\mathrm{NS})$. The $\mathrm{SBRS}_{\mathrm{NSVT}}$ values were significantly higher than the $\mathrm{SBRS}_{\text {vals }}$ values $(12.5 \pm 5$ vs. $9.4 \pm 7$ 
$\mathrm{mmHg} / \mathrm{s}, \mathrm{p}<0.008)$. Nevertheless, these parameters correlated closely $(\mathrm{R}=0.86, \mathrm{p}<0.001)$. Their mean difference was 3.2 $\pm 4.8 \mathrm{mmHg}$. The Bland Altman plot (Figure 4.) also indicated a modest relationship.
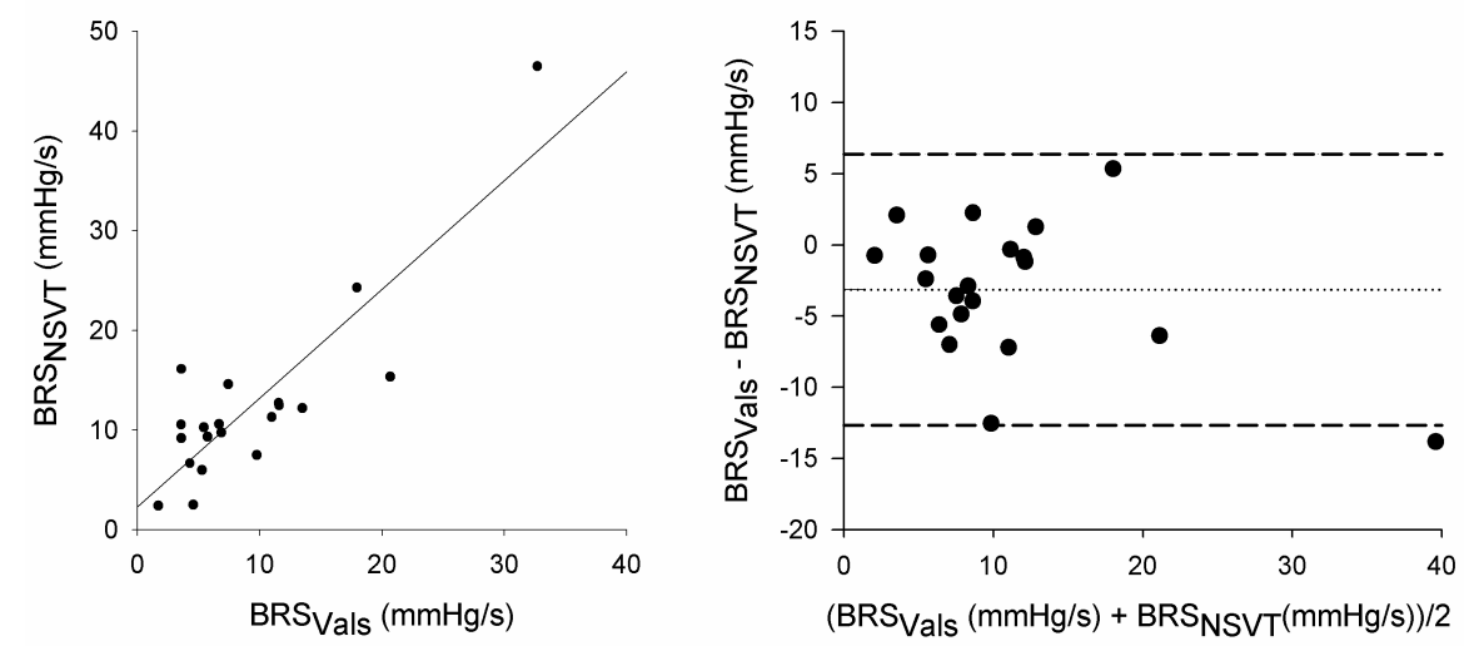

Figure 4. Relationship of $S B R S_{N S V T}$ and $S B R S_{\text {vals }}$ illustrated by linear regression and Bland Altman plot.

With two exceptions MAP ${ }_{\mathrm{TS}}$ could be calculated over the first five post-NSVT cycles. Both $\mathrm{SBRS}_{\text {vals, }}$, and $\mathrm{SBRS}_{\mathrm{NSVT}}$ correlated closely with $\mathrm{MAP}_{\mathrm{TS}}(\mathrm{R}=0.77, \mathrm{p}<0.001$ and $\mathrm{R}=0.86$, $\mathrm{p}<0.001$ respectively).

\subsection{Orthostatic responses of BRScv ad HRT parameters.}

Twelve male subjects were recruited for the study. Their mean age was $61 \pm 11$ years. Indication for permanent pacemaker implantation was intermittent AV block in 11 cases, and carotid sinus syncope in one. In four instances the number of spontaneous BRS up- or down sequences on the patient's baseline recording was less than 5; therefore, these patients were excluded from the further analysis. For the remaining eight subjects, the up-BRS values and the TS values correlated closely both in supine and upright positions $(\mathrm{R}=0.94, \mathrm{P}<0.001$ and $\mathrm{R}=0.96, \mathrm{P}<0.001$, respectively). Close relation was also found between the down-BRS and TS values in the supine and upright positions $(\mathrm{R}=0.92, \mathrm{P}<0.001$ and $\mathrm{R}=0.94, \mathrm{P}<0.001$, respectively). TO and up-BRS negatively correlated both in supine and in upright posture ( $\mathrm{R}$ $=-0.5$ and $\mathrm{R}=-0.66$ ). However, the correlation did not reach statistical significance. Similarly, non-significant negative correlation was found between supine and upright downBRS and TO $(\mathrm{R}=-0.57$ and $\mathrm{R}=-0.64$, respectively). TS and TO were significantly and negatively correlated both in laying and in tilted position $(\mathrm{R}=-0.71, \mathrm{P}=0.05$ and $\mathrm{R}=-0.77$, $\mathrm{P}=0.03$, respectively). Compared to supine, upright tilt resulted in significant $\mathrm{RR}$ interval shortening. The systolic arterial pressure remained unchanged and the diastolic pressure showed non-significant elevation. Both the up- and down-BRS values showed significant decrease upon tilting. Similar significant decline was detected in the TS values (Table 2). The individual postural changes of baroreflex gains and TS values are shown in Figure 5. In contrast, the TO values showed no significant postural decrease (Table 2.). 
To Remove Please Registered With http://www.pdf-converter-software.com

Table 2. Hemodynamic, baroreflex and HRT responses to orthostasis

\begin{tabular}{llll}
\hline & Supine & Upright & $P$ \\
\hline Mean RR interval (ms) & $820 \pm 139$ & $747 \pm 120$ & 0.005 \\
Mean systolic pressure (mm Hg) & $125 \pm 25$ & $128 \pm 18$ & NS \\
Mean diastolic pressure (mm Hg) & $69 \pm 17$ & $76 \pm 19$ & NS \\
Up BRS (ms/mm Hg) & $7.8 \pm 6.9$ & $4.2 \pm 3.7$ & 0.02 \\
Down BRS (ms/mm Hg) & $6.8 \pm 4.9$ & $3.9 \pm 3.3$ & 0.003 \\
TO (\%) & $-2.62 \pm 4.9$ & $-2.15 \pm 2.6$ & NS \\
TS (ms/cycle) & $21.6 \pm 21.5$ & $13.9 \pm 19.6$ & 0.02 \\
\hline
\end{tabular}

Data are given as mean \pm standard deviation
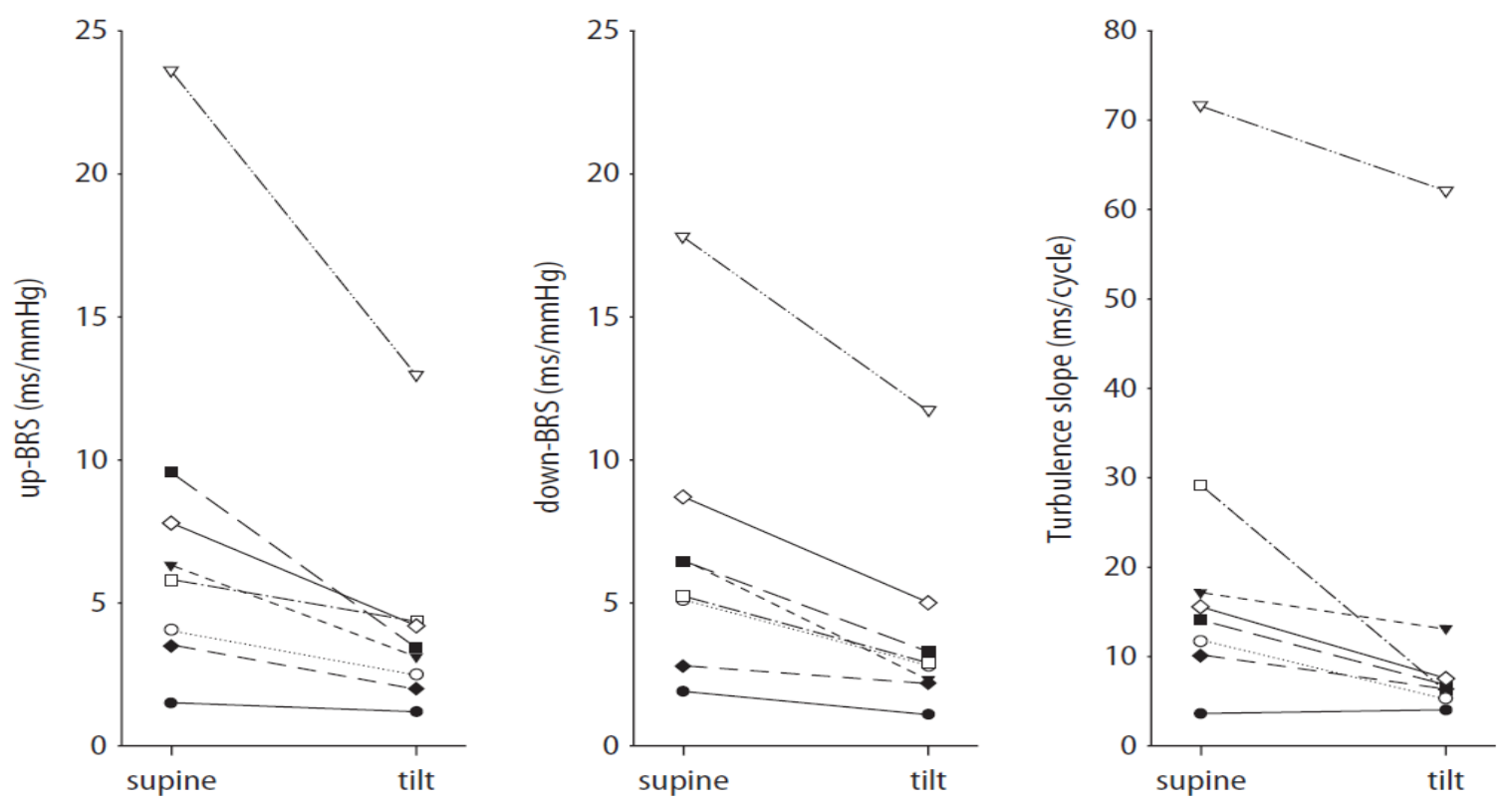

Figure 5. Individual up-BRS, down-BRS, and TS responses upon tilting 


\section{DISCUSSION (INTERPRETATIONS AND CONCLUSIONS)}

\subsection{Observations during carotid artery stenting.}

Swings of the arterial pressure from its resting point elicit immediate counterregulation mediated by arterial baroreflexes. Human baroreceptors are located in the aortic arch and in the two carotid sinuses. It is not surprising that in the course of carotid stent deployment hemodynamic instability - mediated by autonomic nervous mechanisms - occurs. Carotid stenting may be accompanied by hemodynamic instability during or after the procedure. The stent dilation leads to immediate activation of stretch-sensitive mechanoreceptors. This results in a marked, immediate reaction, which may persist postprocedurally. Some publications have given account of cases where no preventive measures were used against bradycardia. In the report of Yadav et al. (1997) balloon inflation induced syncope in four cases; all of them were known to have complete occlusion of the contralateral carotid artery. Teitelbaum et al. (1998) recorded bradycardia and asystole lasting less than three seconds during angioplasty and stent implantation, in a similar population. There are several reports documenting the use preventive measures at the time of carotid angioplasty, to preclude bradycardia response. In two studies - Mendelshon et al. (1998) and Harrops et al. (2001) - the authors performed the procedures with temporary ventricular pacing. The ventricular "escape" frequency was set at $60 /$ minute in both examinations. In these studies the hemodynamic and autonomic regulation might have been affected by the pacemaker itself. At the time of sinus bradycardia, ventricular stimulation could have resulted in atrioventricular asynchrony, complicating the situation by a "pacemaker-syndrome-like effect". This effect may explain why these measures did not prevent syncope in certain cases.

The risk profile of the population in our study was comparable to that of Yadav's and Teitelbaum's. In our study we defined episodes with pauses of three or more seconds as "pathological pauses". Actually both patients who suffered syncope in our study met this criterion. Iv. atropine was given in $29 \%$ of the cases in our study. Transient hypotension, according to our experience, was quite a uniform reaction. Although the magnitude of the blood pressure drop in the subgroup with pathological pauses was greater, significant blood pressure drop was often seen in cases without pauses as well. Transient hypotension seemed to be a harmless phenomenon in our study.

The reduction in BRS indices in our study could be related to the age of the subjects. The inverse correlation between the autonomic markers and the age is well documented. Accompanying diseases such as coronary heart diseases, diabetes mellitus and hypertension may have also influenced the data. Our study evidenced important technical limitations. Frequent rhythm disturbances often precluded even the simple heart rate variability tests, whereas baroreflex sensitivity sequences could be determined in less than half of the patients. The same limitation was seen in one of our other studies: - BRS sequences occurred in insufficient number, in patients suffering from intermittent AV-block.

In conclusion hemodynamic instability accompanying carotid artery dilatation is a transitory and benign phenomenon, which is not predictable from the subjects' baseline baroreflex markers. In elderly patients who suffer from accompanying diseases, the traditional heart rate variability and baroreflex markers reveal universally pathological and low values. 


\subsection{A short comment on arterial baroreflex sensitivity indices.}

The arterial baroreflex system plays a role in stabilizing the arterial pressure. The efferent limbs of the regulation include efferent vagal and sympathetic activation. From the pulse rate responses, induced by arterial pressure changes a baroreflex gain, the cardiovagal BRS could be derived. This BRScv in the cardiological practice and publications is often denoted as "BRS", without specification. This negligency deceives many readers, who believe, that only one kind of baroreflex mechanism, and one kind of baroreflex gain, the "BRS" exists. In fact, even the BRScv values can differ depending on whether they were derived from increasing or decreasing sequences (up- and down BRS). There is no generally accepted definition of sympathetic BRS. BRSsym could be calculated from, the muscle sympathetic nerve activity, or from certain other parameters. It was shown by previous publications and is confirmed by our study that is included into the dissertation, that indices derived from the arterial pressure changes of the Valsalva maneuver (BRSvals), and induced VT (BRSvt) are interrelated and valid forms of BRSsym.

\subsection{New sympathetic baroreflex indices derived from pressure recovery from paced NSVT, and Valsalva maneuver.}

It has been long noted that arterial pressure fluctuation following a premature ventricular contraction resembles to the late phases of the Valsalva. Blood pressure recovery from Valsalva maneuver in patients suffering from autonomic failure varies directly with the severity of adrenergic impairment, and the sluggish blood pressure recovery following premature ventricular contraction is also characteristic of these patients. In spite of these similarities, important differences between the two responses could also be acknowledged. A premature ventricular beat with its compensatory pause results in a temporary decline in arterial pressure, triggering sympathetic response. Conflicting influences could be operational during ventricular tachycardia, which is accompanied by unloading of the arterial, and loading of the cardiopulmonary baroreceptors. Arterial baroreflex control predominates in mediating sympathoexcitation. Following ventricular extrasystole, or NSVT, the sympathetic activation generates arterial pressure elevation. The changes in stroke volume following a longer pause contribute significantly to the restoration of arterial pressure. The vagal withdrawal, which accompanies the temporary hypotension, may also interfere with the MAP recovery profile. Therefore, gains derived from the MAP recovery are not pure indices of sympathetic activity.

During Valsalva maneuver complex interactions of mechanical and reflex responses occur. The arterial baroreflex activation during the early strain phase reflects opposing influences of different aortic and carotid baroreceptors stimulation. With prolonged straining, both the aortic cross sectional area and the peripheral pressure decline. These changes are reflected by increased sympathetic muscle nerve activity. Post-straining arterial pressure elevation is proportional to (and probably caused by) the preceding increase of sympathetic activity. With regard to these discrepancies in their mechanisms, the correlation that we found between the Valsalva-derived and NSVT-derived sympathetic baroreflex indices is remarkably close.

Directly, or indirectly, both vagal and sympathetic systems contribute to the shaping of the post-extrasystolic heart rate pattern. Therefore, it could be assumed that HRT TS provides information about both systems. The two systems, however, can be affected differently by various pathological conditions. Lieshout et al reported misleading Valsalva heart rate responses of patients, who suffered form isolated efferent sympathetic neuropathy. One may 
object that such patients are very rarely encountered in real life, but this is not necessarily true. Contrary to the general belief, the sympathetic control of baroreflexes is often abnormal before parasympathetic dysfunction can be demonstrated in diabetic patients. The independent predictive value of a sympathetic baroreflex gain in large patient populations suffering from cardiac diseases is yet to be determined. By the analogy of HRT TS calculation recent publications have already demonstrated the feasibility of blood pressure turbulence analysis. Since the calculation of systolic-, or MAP slopes are not restricted to the period preceding the baseline crossing, perhaps these parameters provide more thorough information about the dynamics of arterial pressure recovery. Importantly, a trend has been reported toward decreased blood pressure slopes among patients with heart, or with idiopathic dilated cardiomyopathy.

The Valsalva maneuver is dependent on several factors, and requires good cooperation of the subjects. The effects of the subject's position, the magnitude and duration of straining, and the role of breathing pattern before and after the maneuver are well documented. Certain subjects, such as elderly patients with Parkinson's disease are unable to perform the test. The "square wave Valsalva response", which was first described among patients with heart failure, could be recorded in healthy volunteers suffering from no apparent heart disease as well. In spite of these limitations, Valsalva maneuver derived SBRS $_{\text {vals }}$ remains a valuable noninvasive tool in assessing the sympathetic arterial baroreflex regulation.

Arterial pressure recoveries from Valsalva maneuver- and VT-induced hypotension are analogous phenomena. While the Valsalva maneuver is suitable for studies in the general populations, $\mathrm{SBRS}_{\mathrm{NSVT}}$ could be incorporated into the cardiac electrophysiological protocols. Patients with implantable ant arrhythmic devices might be also investigated non-invasively, by external programming of NSVT-s, allowing standardized serial assessment of sympathetic arterial baroreflex regulation

\subsection{Postural influences on BRScv and HRT parameters.}

The capability of HRT parameters to predict adverse outcome was first documented by Schmidt et al in 1999. In their landmark study retrospective analysis was performed on Holter tapes of acute myocardial infarction survivors, who were enrolled in large-scale follow-up studies (MPIP and EMIAT trials). Since 1999 HRT analysis has gained general acceptance in clinical cardiology. Recently, a consensus statement, issued by the International Society for Holter and Noninvasive Electrophysiology (ISHNE) redefined its measurement standards and clinical use. Importantly, in two ongoing investigations, in the REFINE ICD trial and in the ISAR-ICD trial, the benefit of ICD implantation will be tested in high risk patients, defined by (among other selection criteria), abnormal HRT. The results of these trials may dramatically influence the future of HRT analysis.

Regardless the results of the ongoing studies, it remains still important to define the potential modifiers and the limitations of the HRT method. We know that BRScv determinations could be influenced by several factors, including circadian variations, sleepwake differences, and the influences of physical activity, emotions, post-prandial state and posture. Circadian variation of the HRT parameters has been recently also noted. HRT parameters could be also influenced by the subjects' age. Our study adds orthostasis to the list of proven confounders. The parallel changes of TS and BRScv in response to orthostasis further supports their common mechanism. Based on our findings postural factor should be always considered when assessing TS from Holter tapes; for instance, by performing separate analyses of recordings taken in supine and erect positions. 
From the very first description of HRT parameters, the exact mechanism of postextrasystolic RR interval changes has been subject of debate. By now, most of the researchers are convinced, that these RR interval responses are vagally mediated, as it has been shown that atropine administration could abolish them. Numerous studies investigated the relationship between baroreflex and HRT phenomenon. The method of BRS assessment included the phenylephrine BRS test in 2 studies, Valsalva derived BRS in 1 study, cross spectral BRS in 1 and spontaneous sequence BRS in 2 investigations. In 3 studies, the BRS assessment was performed in electrophysiological laboratories, utilizing paced PVC-s. All of the above studies confirmed the baroreflex origin of HRT. Our findings give further support to these observations. Instead of invasive procedure we used non-invasive, externally programmed pacemaker stimuli-a method that was introduced by Raj et al in 2005, in patients with implanted ICD. Our present study confirms that this technique is feasible in assessing HRT in elderly patients with atrioventricular conduction abnormalities.

\section{LIMITATIONS}

The sequence BRS method, used in our studies, has limitations. Firstly, - as indicated by our results, - in many cases the method is unfeasible because of the lack of spontaneous sequences. The lack of spontaneous sequences is more common in certain patient populations, such as the elderly, or those with Parkinson's disease. Secondly, the high correlation limit for the spontaneous sequences may impose a selection bias. In order to mitigate selection bias toward higher BRS values we set no RR interval limit. Thirdly, the validity of spontaneous baroreflex tests is the subject of an ongoing debate. Arterial baroreflex, in real life, acts within a closed loop system. The oscillations of the blood pressure and pulse rate encompass both feedback (baroreflex) and feed forward relations. The traditional human baroreflex tests, such as the vasoactive drug administration, the neck suction test, and the Valsalva maneuver, alter the arterial transmural pressure and briefly open part of the closed loop. In contrast, spontaneous indices, which are based on arterial pressure and heart period changes with no apparent external cause for their occurrence, may errantly suggest baroreflex involvement. Of note, the PVC-induced response may represent another example of "opening the loop." Indeed, the hemodynamic responses to PVCs have a resemblance to that of Valsalva maneuver. Finally, the somewhat different correlations and postural responses of the TS and TO parameters merit explanation. All the traditional BRS tests, (including the methods of spontaneous sequences), utilize "ramp-like" changes in the stretch of arterial baroreceptive areas. The relationship between the stimulus (arterial pressure, neck chamber pressure, or carotid diameter) and the response (RR intervals) is characterized by calculating the gain between these variables. It might be erroneously concluded that baroreflex response requires several cardiac cycles to manifest. In reality, the vagally mediated pulse interval responses are very quick and could be short lasting. These minuscule "single beat'" baroreflex events are usually not assessable with traditional tests, and their relationship to the pressure ramp-related parameters is not yet characterized. TO represent such brief baroreflex manifestation and in this capacity is a unique parameter.

\section{RESPECTIVE FINDINGS AND FUTURE CLINICAL IMPLICATIONS}

Our observations indicate that BRS and HRV values are generally depressed in elderly arteriosclerotic population, allowing no real prediction of bradycardia upon carotid artery stenting. These observations are supported by recent clinical studies. On the other hand our study confirmed, that the episodes of intervention related transient bradycardia is generally 
benign, requiring no temporary pacemaker insertion. This finding may help to avoid unnecessary costs and complications of the procedures.

Our observation of the close association between BRScv and HRT indices are in line with the results of previous studies. The relationship is emphasized by the parallel behavior of these indices upon orthostasis. The latter finding may help to explain the documented daynight differences of HRT parameters, derived from Holter tapes. Besides changes resulting from wake-sleep differences, the postural decline of vagal heart rate regulation should be also considered. The results also exemplify the uncertainties arising from Holter recording based studies. In our publication we concluded that HRT parameters could be more reliably assessed during controlled laboratory conditions. We have even suggested that external induction of arrhythmia via programming implanted devices may serve as a tool for longitudinal studies in certain patient populations. Since the publication of our paper, studies have been started with similar rationale. In the ongoing DECIDE HF study (http://clinicaltrialsfeeds.org/clinicaltrials/show/NCT00949676) HRT is calculated by implanted devices after spontaneous PVC-s or after device stimuli.

Our study on the arterial pressure recovery from paced NSVT-induced pressure decline indicate, that this maneuver can serve as a tool in characterizing sympathetic baroreflex regulation. The indices - derived from induced NSVT - are closely parallel to an other, validated sympathetic gain, which is derived from the pressure responses of Valsalva maneuver. Confirmation of the converging nature of these techniques may allow uniformization of the methods used in healthy and sick populations. 
To Remove Please Registered With http://www.pdf-converter-software.com

\section{ACKNOWLEDGEMENTS}

I would like to express my deep and sincere gratitude and my honest respect to my supervisor, Prof. Dr. László Rudas for supporting me throughout those years since 1995 in my clinical and scientific work and my dissertation.

I would like to thank Prof. Dr. Tamás Forster for giving me the opportunity to conduct my studies alongside my clinical responsibilities, and for all of my co-workers who helped me in the research work.

Last but not least I would like to express my heartfelt gratitude to my wife and my whole family for their support, help and tolerance, and also for my daughter, Emma for all kind of help she could give me. 